\title{
Patients' preferences for primary health care - a systematic literature review of discrete choice experiments
}

\author{
Kim-Sarah Kleij ${ }^{*} \mathbb{D}$, Ulla Tangermann, Volker E. Amelung and Christian Krauth
}

\begin{abstract}
Background: Primary care is a key element of health care systems and addresses the main health problems of the population. Due to the demographic change, primary care even gains in importance. The knowledge of the patients' preferences can help policy makers as well as physicians to set priorities in their effort to make health care delivery more responsive to patients' needs. Our objective was to describe which aspects of primary care were included in preference studies and which of them were the most preferred aspects.
\end{abstract}

Methods: In order to elicit the preferences for primary care, a systematic literature search was conducted. Two researchers searched three electronic databases (PubMed, Scopus, and PsycINFO) and conducted a narrative synthesis. Inclusion criteria were: focus on primary health care delivery, discrete choice experiment as elicitation method, and studies published between 2006 and 2015 in English language.

Results: We identified 18 studies that elicited either the patients' or the population's preferences for primary care based on a discrete choice experiment. Altogether the studies used 16 structure attributes, ten process attributes and four outcome attributes. The most commonly applied structure attribute was "Waiting time till appointment", the most frequently used process attribute was "Shared decision making / professional's attention paid to your views". "Receiving the 'best' treatment" was the most commonly applied outcome attribute. Process attributes were most often the ones of highest importance for patients or the population. The attributes and attribute levels used in the discrete choice experiments were identified by literature research, qualitative research, expert interviews, or the analysis of policy documents.

Conclusions: The results of the DCE studies show different preferences for primary health care. The diversity of the results may have several reasons, such as the method of analysis, the selection procedure of the attributes and their levels or the specific research question of the study. As the results of discrete choice experiments depend on many different factors, it is important for a better comprehensibility of the studies to transparently report the steps undertaken in a study as well as the interim results regarding the identification of attributes and levels.

Keywords: Preferences, Primary health care, Discrete choice experiment, Systematic review

\footnotetext{
* Correspondence: Kleij.Kim-Sarah@mh-hannover.de

Institute for Epidemiology, Social Medicine and Health Systems Research,

Hannover Medical School, Carl-Neuberg-Str. 1, 30625 Hanover, Germany
} 


\section{Background}

According to the World Health Organization's (WHO's) declaration of Alma-Ata, primary care is a key element of health care systems. As it addresses the main health problems in the community and often serves as the patients' first level of contact with the health care system, primary care is highly important to all citizens [1]. Primary health care encompasses different curative and preventive services, such as diagnosis and treatment of chronic and acute conditions, and patient education concerning the major health problems. Furthermore, primary care traditionally is health care which, in the first place, focuses on the needs of the patients [2]. As most of the Western populations are continuously aging and, hence, the burden of chronic conditions is increasing, primary care even gains in importance. The knowledge of the patients' preferences can help policy makers as well as physicians to set priorities in their effort to make health care delivery more responsive to patients' needs [3]. Moreover, the patients' satisfaction positively influences their compliance with the treatment [4]. As many countries face a shortage of general practitioners (GPs) in rural and remote areas, the maintenance of an adequate primary care provision is a central task of health care systems and therefore a highly important subject for health policies. In order to guarantee an adequate, needs-based medical supply strategies, such as new and innovative models of care are needed. Philips et al. [5] for example state that in many European countries an increasing number of patients is using emergency rooms for less urgent problems in out-ofhours situations. If policy makers attempt to address these problems and to reorganize out-of-hours care more efficiently, they need to know patients' preferences. If the reorganization does not take into account the patients' needs and preferences, patients probably would not use them and continue visiting emergency rooms. Thus, it is of high relevance for the future organization of primary care and the introduction of new models of care to know the population's preferences for different aspects of primary care.

Preferences for medical care can be defined as "statements that indicate the importance of specific aspects of clinical behavior of care providers or the organization of care" ([3], p. 1573). They indicate what should happen and they differ from the concepts of expectations, experiences and satisfaction. The latter can be described as the assessment of the care received, i.e. the assessment of the experiences [2]. Expectations are determinants of satisfaction as well. While predictive expectations describe what people actually believe will happen in the future, ideal expectations are desires connected to an idealistic state of beliefs [6]. Thus, ideal expectations are more abstract than preferences.
The discrete choice experiment (DCE) is a common technique to elicit preferences for health care services or technologies. DCEs are based on Lancaster's [7] theory according to which the utility of goods or services is determined by different characteristics, called attributes, that characterize the good or service. Each attribute has different specifications, so called attribute levels. In a DCE, a good or service is described based on changing combinations of attribute levels and participants are asked to choose out of two or three different options the one, they prefer. The choices over a number of alternatives can then be analyzed to calculate the relative importance of the attributes. It is assumed that respondents take into account all information provided and then select the alternative which provides the highest utility to them [7]. Changes in the attribute levels can alter the preferred choice alternative of participants [8]. In addition to Lancaster's theory, discrete choice experiments are based on the random utility theory (RUT) $[9,10]$. In contrast to the classic consumer theory, the RUT states that individual choice behavior is probabilistic rather than deterministic. Thus, the utility of a good or service can be divided into an explicable, systematic component and a nonexplicable, random component. The latter can for example be due to unobserved preference variation or measurement error, [11].

There are several discrete choice experiments focusing on patient or public preferences for primary care and GPs. Those DCEs include attributes like "Waiting time till appointment" or "Length of the consultation". The levels for the attribute waiting time could be "same day", "one day" and "two days", for example. If "Price" is one of the attributes of the DCE, it is possible to calculate the willingness to pay for the other attributes. As a DCE is an attribute-based method of measuring preferences, the identification of appropriate attributes is an essential task [2]. Attributes should be important to the respondents on the one hand and relevant to policy makers on the other hand [12]. Therefore, the identification of attributes should always be supported by evidence derived from the literature and/or from qualitative research [13].

The aim of this systematic review is (1) to provide an overview of the attributes and attribute levels used in the discrete choice experiments and of how they were selected and (2) to reveal which attributes of primary care are most important for patients and the population.

\section{Methods}

\section{Eligibility criteria and search strategy}

We conducted a systematic review of published studies reporting stated preferences, particularly discrete choice experiments for primary health care in OECD countries (see Additional file 1 for the review protocol). For obtaining 
a recent overview of the literature, we searched for studies published in the last 10 years (2006-2015). Electronic searches of relevant databases were conducted on December 7th 2015. The search included (1) terms related to primary care and (2) terms related to preferences and discrete choice experiments, respectively. Search terms were: ("patient" preference" " OR "patient" priorities" OR "public preference" "conjoint analysis" OR "stated preference") AND ("primary care" OR "general practitioner" $\mathrm{OR}$ "GP" OR "family doctor" OR "family physician"” OR "family medicine"”) (see Additional file 2 for a detailed presentation of the search strategy). The search was conducted in the databases: PubMed, Scopus and PsycINFO; further literature was added from an additional hand search in the reference lists of the included articles. Titles, abstracts and full-texts of the identified studies from the search strategy were screened for relevance by two of the authors independently (KSK, UT) and ambiguous cases were discussed.

\section{Inclusion and exclusion criteria}

Discrete choice experiments were included if they were published in English and if either the population or patients were asked about their preferences for different aspects of primary health care in general were included. There were no constraints about age, sex or origin of the participants. All types of survey methods were included: face-to-face, telephone, postal, and online surveys. Furthermore, there were no limitations according to the aspects or attributes of primary care, as long as they were relevant for medical care.

Studies were excluded if they only focused on specific conditions because the objective of the literature review was to cover a broad range of attributes that are relevant to all patients, not only patients with specific diseases. Furthermore, we excluded studies that exclusively focused on end-of-life or palliative care or on shared-decision making because they do not refer to primary care in general but focus on an issue that we think is only one aspect or attribute of primary health care.

\section{Outcomes and comparison of results}

The purpose of this review was to identify the attributes and attribute levels used in DCE studies and to provide an overview of the aspects of primary care which are most important to patients and the population. To that end, we divided the identified attributes into the three dimensions "structure", "process" and "outcome". These dimensions are based on Donabedian's model for quality of health care $[14,15]$ and are appropriate to group the wide range of primary care attributes and to have a closer look on what dimensions of health care are most important for the respondents when choosing primary care conditions. The dimension "structure" refers to objective parameters such as material resources, personal resources and organizational structure. "Process" includes all activities taking place while giving and receiving care, such as diagnosis, prescription and interpersonal aspects. The dimension "outcomes" denotes the effects of health care delivery, including improvements in the patients' knowledge and changes in their behavior, on the health status of patients [15].

Beyond that, we aimed at giving an overview of how the attributes and attribute levels were selected by the researchers and why they were determined to be relevant. In general, attributes used in a DCE should cover a range that may be relevant to subjects, even if the levels are hypothetical [13]. According to Lancsar and Louviere [11] or Bridges et al. [13] it is good research practice if inclusion or exclusion of potential attributes and levels is based on literature reviews and qualitative research, such as focus group discussions or semi-structured interviews with samples of relevant persons and/or experts. Therefore, we examined if the selections of attributes were based on one or several of these techniques. To extract the data and compare the results, we tabulated the findings for each study.

\section{Results \\ Study selection}

The search strategy resulted in 1515 findings (Fig. 1): 827 through Scopus, 482 through PubMed, 201 through PsycINFO, and five through the additional hand search. Four hundred fifty-five duplicates were removed and after the subsequent screening of titles, abstracts and full-texts, 19 publications met the inclusion criteria and were thus included in our analysis. ${ }^{1}$ Most studies were conducted in England [16-24]. Other studies were conducted in Scotland [25, 26], Italy [27, 28], Denmark [29], Sweden [30], and the United States [31, 32]. One multinational study [33] was conducted in Germany, United Kingdom and Slovenia. A further study [4] did not indicate the country; it was conducted in a 'Western European city'. Most of the publications focus on primary care consultations in general. Others consider out-of-hours services, appointment bookings or the role of prescribing pharmacists. One study examines nurseled versus doctor-led primary care and another publication focuses on retail clinics.

\section{Study and sample characteristics}

Table 1 summarizes the study and sample characteristics. Participants were patients in ten of the articles and the population in eight of the studies. About three quarters of studies used self-complete discrete choice experiments and the number of attributes varied from three to seven, with a mode of four attributes $(n=6$ 


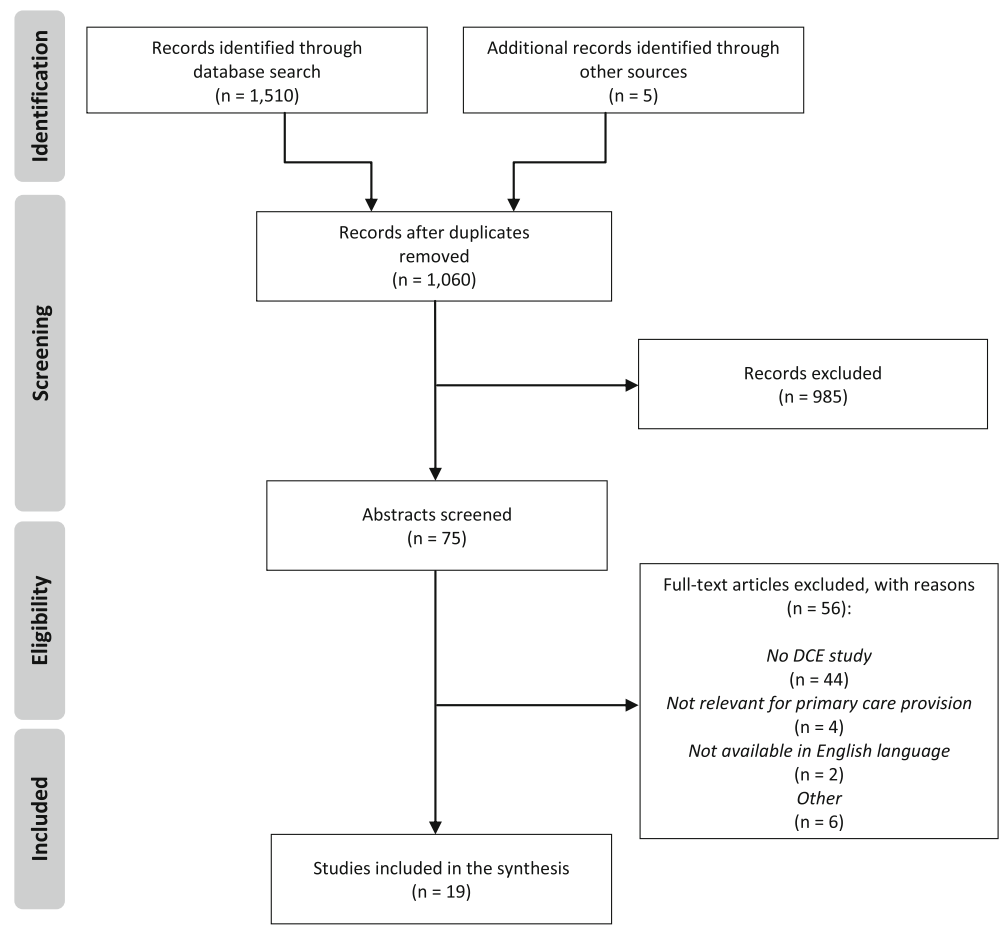

Fig. 1 Flow diagram

studies). The respondents were faced with a maximum of eight different choice sets in most surveys. The sample size was $<500$ in six and $>1000$ in seven of the studies. Eight studies had a response rate between $40 \%$ and $60 \%$. Three studies reported a response rate of less than $40 \%$ and two studies of more than $60 \%$. In these two studies $[17,33]$ the questionnaires were handed out in a general practice. While one was a self-complete questionnaire, the other was an interviewer-administered survey. Furthermore, some of the studies tested for the influence of sociodemographic and disease-specific characteristics on the preferences $[17,19,23,25,28,31]$.

\section{Attributes and levels of primary care}

A crucial aspect of discrete choice experiments is the selection of attributes and associated levels that adequately outline the health service a study focuses on. As there are no accurate rules to determine attributes and levels presented in a DCE, we examined how they were selected by the researchers.

Table 2 lists the different methods used by the researchers to determine the appropriate attributes and attribute levels. The most frequently used methods, which are i.a. recommended in the Conjoint AnalysisChecklist published by the International Society for Pharmacoeconomics and Outcomes Research (ISPOR), are a literature research or a systematic literature review ( $n=10$ studies) and qualitative research, such as focus groups or semi-structured interviews ( $n=8$ studies).
Other options to identify attributes are discussions with experts $(n=3)$, an analysis of policy documents $(n=2)$ or selection of attributes based on attributes used in former studies $(n=3)$. There are some studies that used several of these methods to identify relevant attributes. Gerard [17] for example conducted seven semi-structured interviews with general practice patients and searched for policy documents and literature to define attributes and levels. In addition to a literature search [2] Cheraghi-Sohi et al. [16] performed qualitative research in the form of the 'think aloud' technique to verify the comprehension of the potential attributes. Overall, 11 studies (61.1\%) used two or more of these methods to identify attributes and attribute levels (mostly literature review and qualitative research), five studies (27.8\%) used one of these methods and two did not report on the identification of attributes.

Table 3 provides an overview of all attributes included in the identified studies and of how many studies used them. As previously described, the attributes are clustered into the dimensions "structure", "process" and "outcome". Overall the studies used 18 different structure attributes, 10 process attributes and 3 outcome attributes. Summed up, 52 structure attributes, 27 process attributes and 4 outcome attributes were used. That makes 2.9 structure attributes, 1.5 process attributes and 0.3 outcome attributes per study, on average. The most commonly used structure attribute was "Waiting time till appointment". Other attributes of this dimension which were frequently used are "Care 
Table 1 Study and sample characteristics

\begin{tabular}{ll}
\hline & Number of studies (\%) \\
\hline Sample & $10(55.6 \%)$ \\
Patients & $8(44.4 \%)$ \\
Population & \\
Administration of survey & $13(72.2 \%)$ \\
Self-complete questionnaire & $3(16.7 \%)$ \\
Interviewer administered & $2(11.1 \%)$ \\
Computerized interview & \\
Number of attributes & $3(16.7 \%)$ \\
3 attributes & $6(33.3 \%)$ \\
4 attributes & $3(16.7 \%)$ \\
5 attributes & $5(27.8 \%)$ \\
6 attributes & $1(5.6 \%)$ \\
7 attributes & \\
Number of choice tasks per respondent & $12(66.7 \%)$ \\
8 or less choices & $4(22.2 \%)$ \\
$9-16$ choices & $2(11.1 \%)$ \\
Not reported & \\
Sample size & $5(44.4 \%)$ \\
R 500 & $2(11.1 \%)$ \\
> 1000 & $5(27.8 \%)$ \\
\hline &
\end{tabular}

provider/care setting" $(n=8)$, and "Waiting time in the practice" $(n=6)$. Among the process attributes, the one most commonly used was "Shared decision making / professional's attention paid to your views" $(n=6)$. Only a limited number of studies applied outcome attributes. "Chance of receiving the 'best' treatment" was used twice and "Chance contact relieves anxiety" as well as "Likelihood of having illness cured" were each used in one

Table 2 Methods to identify attributes and attribute levels

\begin{tabular}{lll}
\hline Identification method & No. of studies (\%) & \\
\hline Literature research/review & 12 & $(66.7 \%)$ \\
Qualitative research & 10 & $(55.6 \%)$ \\
Other studies & 2 & $(11.1 \%)$ \\
Policy documents & 2 & $(11.1 \%)$ \\
Experts (GPs) & 3 & $(16.7 \%)$ \\
Nothing reported & 2 & $(11.1 \%)$ \\
\hline
\end{tabular}

Sum is greater than 18 and percentage does not sum up to $100 \%$, because some studies used multiple methods to identify attributes and levels
Table 3 Dimensions and attributes

\begin{tabular}{|c|c|}
\hline & $\begin{array}{l}\text { No. of studies } \\
\text { using attribute }\end{array}$ \\
\hline \multicolumn{2}{|l|}{ Structure attributes $(n=18)$} \\
\hline Waiting time till appointment & 12 \\
\hline Care provider / Care setting & 8 \\
\hline Waiting time in the practice & 6 \\
\hline Opening hours & 4 \\
\hline Price & 4 \\
\hline Convenience of the appointment & 3 \\
\hline Individual choice of GP / care provider & 2 \\
\hline Technical equipment / Diagnostic facilities & 2 \\
\hline Waiting time on the telephone & 2 \\
\hline Distance to practice & 1 \\
\hline $\begin{array}{l}\text { How well practice knows services in } \\
\text { neighborhood }\end{array}$ & 1 \\
\hline Informed of expected waiting time & 1 \\
\hline Knowledge of how to access the service & 1 \\
\hline Method of payment & 1 \\
\hline Practice meets specific health needs & 1 \\
\hline Price for the drug & 1 \\
\hline Time spent travelling and waiting & 1 \\
\hline Type of contact & 1 \\
\hline \multicolumn{2}{|l|}{ Process attributes $(n=10)$} \\
\hline $\begin{array}{l}\text { Shared decision making / professional's } \\
\text { attention paid to your views }\end{array}$ & 6 \\
\hline $\begin{array}{l}\text { Continuity of health professional / physician's } \\
\text { knowledge of the patient }\end{array}$ & 5 \\
\hline $\begin{array}{l}\text { Information and explanation (on medicines / } \\
\text { treatment / problem) }\end{array}$ & 5 \\
\hline Length of consultation & 5 \\
\hline Biopsychosocial perspective & 1 \\
\hline Doctor listens & 1 \\
\hline Help offered by professional & 1 \\
\hline Physician's interpersonal manner & 1 \\
\hline Scope of health review & 1 \\
\hline Thoroughness of physical examination & 1 \\
\hline \multicolumn{2}{|l|}{ Outcome attributes $(n=3)$} \\
\hline Receiving the 'best' treatment & 2 \\
\hline Chance contact relieves anxiety & 1 \\
\hline Likelihood of having illness cured & 1 \\
\hline
\end{tabular}

One study [31, 32] used the attribute "acuteness". This attribute does not match one of the dimensions and is therefore excluded

study. The levels of the mentioned primary care attributes vary widely. Some attributes like "Care provider" have quite similar levels across the DCE studies (doctor versus nurse practitioner or practice nurse or primary care team), other attributes like "Waiting time till appointment" have very diverse levels (same day, 1 day, 
2 days, 3 days, 4 days....10 days). All levels and associates attributes are shown in Table 4.

\section{Preferences for primary health care}

The 18 studies are based on a wide range of attributes concerning primary health care. As diverse as the used attributes, so are the results of the preferences measures. Table 4 gives an overview of the study objectives, attributes, attribute levels and the most important attribute in each DCE (Table 4).

Based on the results of the regression analyses conducted in the identified studies the attribute "Care provider" was the most important in 4 of the DCEs $[17,18,25,28]$. Caldow et al. [25] for example show that it is most important for respondents to see a GP rather than a practice nurse. This is even more important than "Continuity of health professional", "Waiting time till appointment", "Likelihood of having illness cured" and "Length of consultation". ${ }^{3}$ Furthermore, 3 studies identified "Shared decision making" as being most important to the respective participants $[19,20,30]$. Hjelmgren and Anell [30] state that having influence on the decision of the care they receive is most important for respondents, followed by "Individual choice of GP/care provider", "Waiting time till appointment" and "Price". Other studies show that "Waiting time" is the most important attribute when choosing a primary care alternative $[3,22,29,32]$. In their study Pedersen et al. [29] identify the typical waiting time until appointment (for routine tasks) to be most important for patients, for instance. This attribute is more important than "Distance to the practice", "Waiting time on the telephone" and "Length of consultation". The other discrete choice experiments determine "Information and explanation (on medicines/ treatment/problem)" [4, 27], "Continuity of health professional/physician's knowledge of the patient" $[24,28]$, "Receiving he 'best' treatment" [26, 33], "Thoroughness of physical examination" [21] and "Individual choice of GP/care provider" [23] as the most important attribute.

\section{Discussion}

Overall, this systematic review of preference studies has identified a considerable number of attributes which effect the organization of primary health care. Moreover, the results of the studies show different preferences for primary health care. Overall 8 studies identify a structure attribute and 8 studies a process attribute as being most important for the respondents. In 2 studies the most significant attribute is an outcome attribute. However, it should be noted that those are absolute numbers, not relative ones. As there are 4 studies that use only structure attributes, all in all there is a lower chance for a process attribute to be the most important one. In addition, there are only 4 studies that include an outcome attribute, so that the chance for those ones to be most significant is even lower. Relative to the number of studies including the attribute dimension in question, a process attribute is most often the one of highest importance, i.e. the one with the highest $\beta$-coefficient in the regression analysis. ${ }^{4}$

In general, the selection of attributes strongly depends on the aim of a study. If a study for example focuses on preferences for appointment booking systems or out-of-hours services, it is evident that the study uses rather structure attributes than process ones. However, what is remarkable is that only a few studies use outcome attributes. Those using outcome attributes have quite different study objectives, such as models of primary care or primary care consultations in general. In two of the four studies that use an outcome attribute it turns out to be the most important one. In both studies it is the same attribute, which is "Receiving best treatment".

Comparing the attributes used in the studies and their results there does not seem to be a pattern according to the studys' objective or origin. The diversity of the results may have different other reasons: The study results may particularly be biased for example by (1) the specific research question of the study or aim of the study or by (2) the selection procedure of the attributes and their levels.

Primarily, the chosen attributes and, thus, the preferences elicited by the DCE depend on the specific research question. Even if the study objective is the same, the precise issue might differ. Pedersen et al. [29] and Turner et al. [24] for example both aim to assess primary care consultations in general, but while the first assess preferences regarding different organizational characteristics, the latter estimate the relative importance of continuity of care compared to other aspects of primary care. Therefore, unsurprisingly, these two studies obtain different results regarding the preferences for primary health care. Pedersen et al. find the attribute "Waiting time" as being the most important one and Turner and colleagues ascertain the process attribute "Information and explanation" to be most significant. Their different research questions may cause a different selection of attributes and consequently different results although the study objectives are the same. In this context, a replication study using the same research question, the same attributes and levels as an existing DCE but comparing different regions and/or populations would be a useful addition to the literature.

Most of the studies followed the recommendations of for example the ISPOR or other authors concerning the selection procedure of the attributes and levels, which 


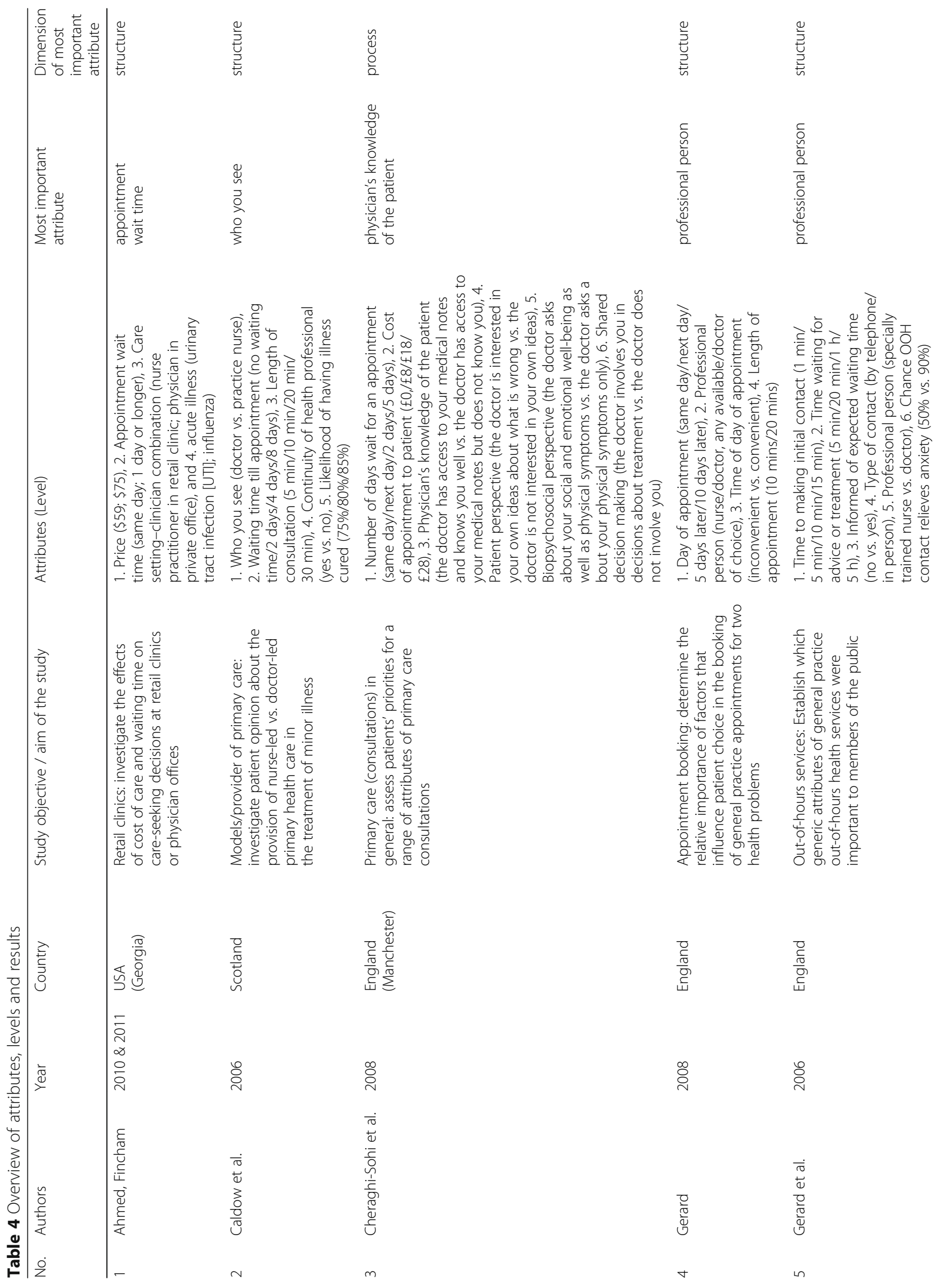




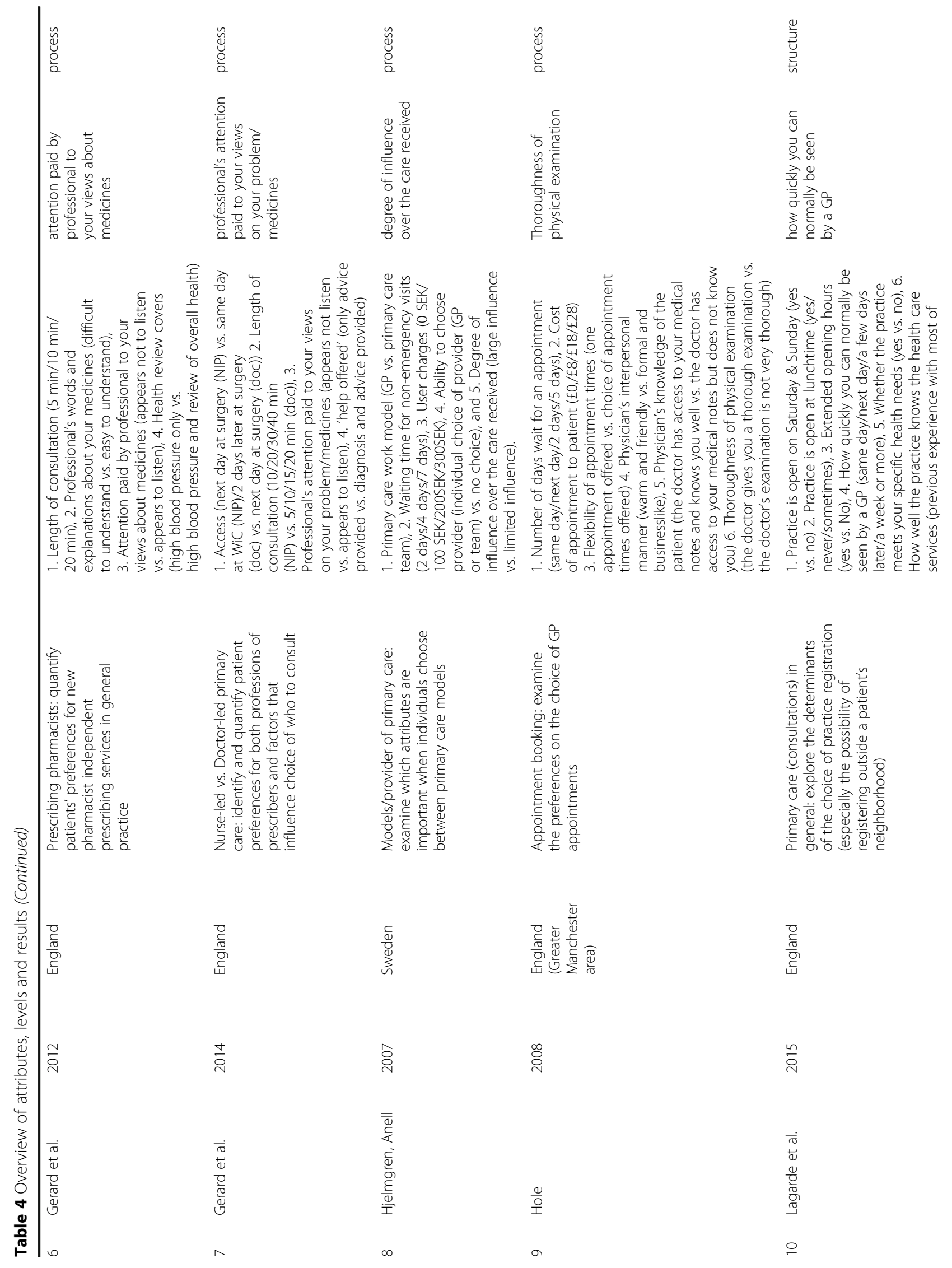




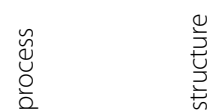

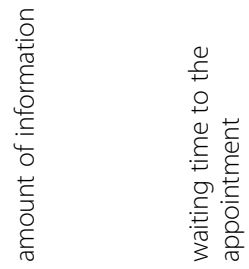

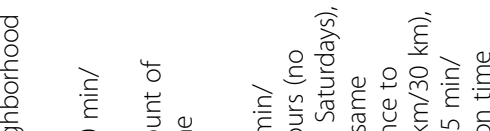

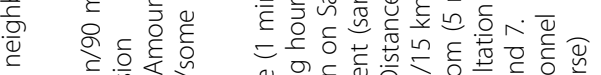

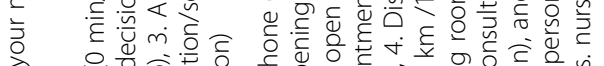

ᄃ

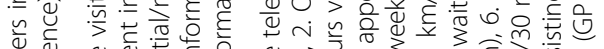

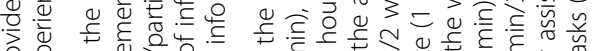

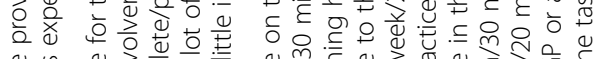

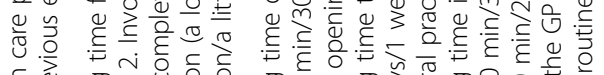

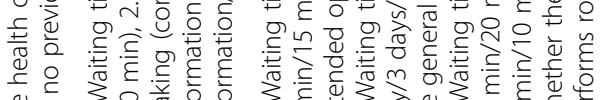

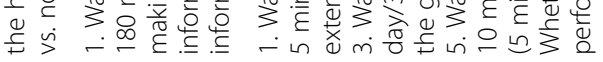

$\frac{\widetilde{\circlearrowright}}{\stackrel{0}{0}}$



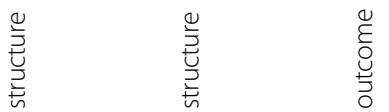

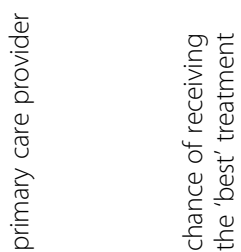

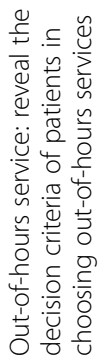

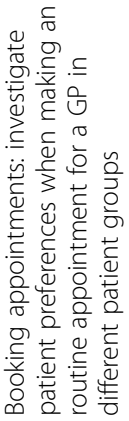
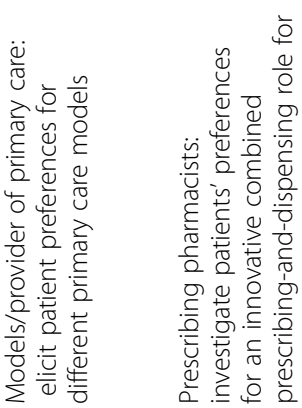

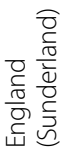

ஓ̊

$\frac{\sqrt{\frac{0}{3}}}{\frac{0}{\frac{0}{0}}}$

$\Xi$

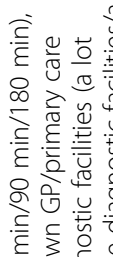

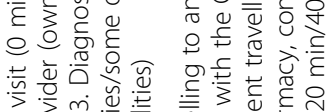

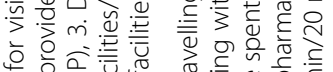


을 严

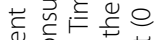
吃

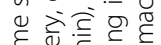

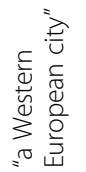

ำ

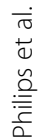

m

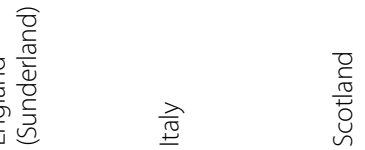

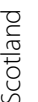

ஓ

$\dot{0}$
$\frac{0}{ \pm}$
$\overline{\overline{0}}$
$\stackrel{\overline{0}}{=}$

$\underline{\bullet}$ 
$\stackrel{0}{0}$
பั
ป<smiles>[Ca]=[Ge]</smiles>

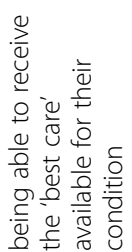

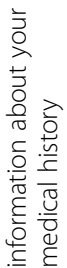

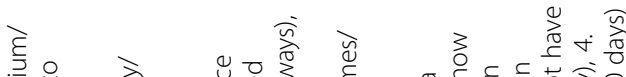

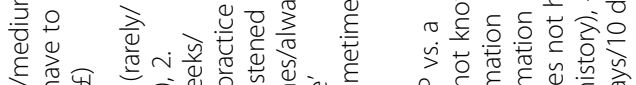

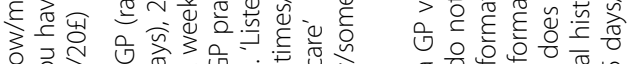
向 पे

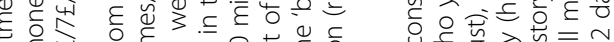

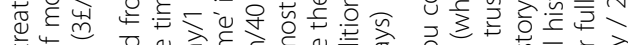

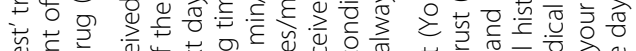

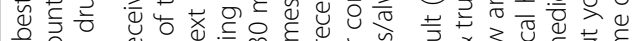
\&

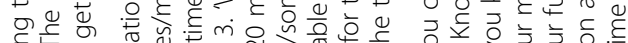
가의

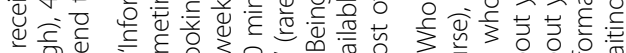

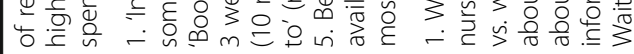
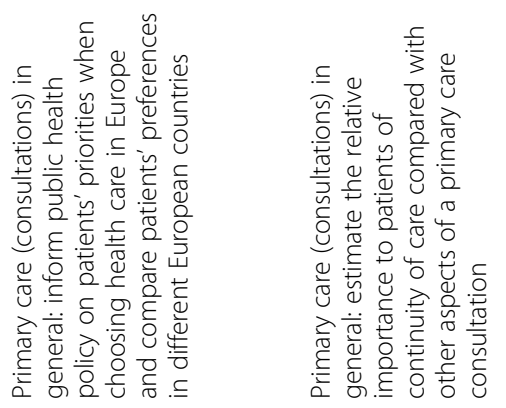

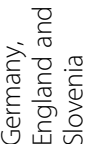

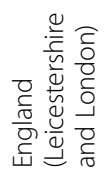

$\stackrel{\triangleright}{\check{D}}$



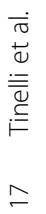

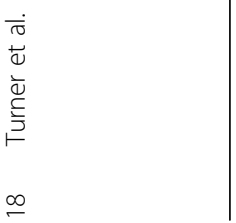


should be supported by evidence like literature reviews, qualitative research or other scientific methods. Although the process of selecting the relevant attributes is highly important for a preference study, there are no precise guidelines on how to translate the literature search or the previous qualitative work into the final attributes and their levels [34]. Amaya-Amaya and colleagues [8] state that an adequate set of attributes and choice contexts, in combination with variation in the attribute levels, is necessary. How this is composed remains unclear in the majority of publications and might be part of the interpretation of the researcher. Only one third of the identified studies claimed to use literature research to identify the relevant attributes and eight studies used qualitative studies. Four studies did not report on the identification of attributes and attribute levels at all. This indicates that not all studies meet the requirements of good research practice, named in different guidelines for conjoint analysis or DCE $[11,13,35]$.

\section{Limitations}

This review of the literature has some limitations. First, the results of a DCE can be influenced by reasons other than the research question or the attribute selection, which cannot all be discussed in this article. Those reasons might be the inclusion of an opt-out option [11, 36] or the description of the scenario. Gerard [17] for example included a "high worry scenario" and a "low worry scenario" in the Discrete Choice Experiment and the results show that seeing a doctor of choice (compared with nurse) is more important if patients are in a chronic, high worry condition than in an acute, low worry condition. Another factor that can influence the results of a DCE is the range of attribute levels. If the attribute "Waiting time until appointment" for example varies between no waiting time, 1 day and 2 days the DCE would - all other attributes and levels equal - probably lead to different results if the levels of this attribute were 1 day, 3 days and 5 days. Attribute levels should be chosen as realistically as possible and extreme values should be avoided [13, 35].

Furthermore, due to the exclusion of non-English language articles, some relevant studies might not have been included in this review. Furthermore, it is possible that the use of other search terms would have led to other search results.

Finally, because the results of a DCE depend on various factors such as research question, selection of attributes and levels and also on method of data analysis, it is not possible to directly compare their results.

\section{Conclusion}

This review gives an overview over the attributes used in DCE studies that measure preferences for primary care.
The results of discrete choice experiments are quite diverse and difficult to compare with each other, because they depend on many different factors, such as the research question, the process of selecting relevant attributes or the method of analysis. In order to achieve a high comprehensibility it is important to transparently report all steps undertaken in a DCE as well as the interim results - especially of the literature research and the qualitative pilot study. This could, for example, be done by publishing the results of the literature search or the qualitative work, or by adding them to an (electronic) appendix. As the final selection of the attributes out of the range of all possible attributes is inherently less transparent and rather interpretative and driven by different interests, the prior steps should be documented as clearly and reproducibly as possible.

Furthermore, this review can be helpful for researchers planning to conduct a DCE in the field of primary health care because it gives a broad overview of attributes and levels used in DCEs in the past 10 years. It also highlights which attributes and dimensions of care provision are important to patients.

Although the results of the DCE studies are not directly comparable, DCEs generally give relevant information on patient preferences within the context of the study setting and can support political decisions that take into account the patients' perspective.

\section{Endnotes}

${ }^{1}$ Please note that there are two publications that base on the same DCE and present the same results. Although we identified 19 publications, we consider only the results of 18 DCE studies.

${ }^{2}$ The attribute "Care provider/Care setting" only refers to the choice of a medical doctor versus a nurse practitioner or practice nurse. It does not relate to the individual choice of a known versus unknown GP.

${ }^{3}$ It must be noted that "Care provider" (being seen by a GP rather than by a nurse) is more important than a unit change in the continuous attributes "Waiting time", "Likelihood of having illness cured" and "Length of consultation"; which means more important than 1 day reduction in waiting time, $1 \%$ change in chance of having illness cured and $1 \mathrm{~min}$ increase in the length of consultation.

${ }^{4}$ For an overview of attributes identified in qualitative studies on preferences for primary care, see for example Cheraghi-Sohi et al. [2].

\section{Additional files}

Additional file 1: Systematic Review Protocol. (DOC $31 \mathrm{~kb}$ )

Additional file 2: Search strategy. (DOC $34 \mathrm{~kb}$ ) 


\section{Abbreviations}

DCE: Discrete choice experiment; GP: General practitioner; ISPOR: International Society for Pharmacoeconomics and Outcomes Research; OECD: Organization for Economic Co-operation and Development; RUT: Random utility theory; WHO: World Health Organization

\section{Acknowledgements}

Not applicable.

\section{Funding}

Kim-Sarah Kleij and Ulla Tangermann were funded by the Federal Ministry of Education and Research (Funding number: 01EH1201B). The funding source had no role in the study and no influence on data collection and analyses, interpretation of results or writing of publications.

\section{Availability of data and materials}

Not applicable.

\section{Authors' contributions}

KSK contributed to the development of the study design, carried out the literature search and analysis, drafted and improved the manuscript, approved the final version and agreed to be accountable for his contribution. UT reviewed the literature search and analysis, and reviewed and commented on the preliminary drafts and final version of the paper, approved the final version and agreed to be accountable for her contribution. VEA reviewed and commented on the preliminary and final paper drafts paper, approved the final version and agreed to be accountable for his contribution. CK contributed to the development of the study design, reviewed and commented on the literature search and analysis, and also reviewed and commented on preliminary paper drafts and the final version paper, approved the final version and agreed to be accountable for his contribution.

\section{Ethics approval and consent to participate}

Not applicable. This is a systematic literature review.

\section{Consent for publication}

Not applicable. This is a systematic literature review.

\section{Competing interests}

The authors declare that they have no competing interests.

\section{Publisher's Note}

Springer Nature remains neutral with regard to jurisdictional claims in published maps and institutional affiliations.

Received: 8 November 2016 Accepted: 5 July 2017

Published online: 11 July 2017

\section{References}

1. WHO. Declaration of Alma-Ata. International conference on primary health care. Alma-Ata, USSR: World Health Organization. 1978,6-12. http://www. who.int/publications/almaata_declaration_en.pdf. Accessed 02 Mar 2017.

2. Cheraghi-Sohi S, Bower P, Mead N, et al. What are the key attributes of primary care for patients? Building a conceptual 'map' of patient preferences. Health Expect. 2006;9:275-84.

3. Wensing $M$, Jung HP, Mainz J, et al. A systematic review of the literature on patient priorities for general practice care. Soc Sci Med. 1998;47(10):1573-88.

4. Kincey J, Bradshaw P, Ley P. Patient satisfaction and reported acceptance of advice in general practice. J R Coll Gen Pract. 1975;25:558-66.

5. Philips $H$, Mahr D, Remmen $R$, et al. Predicting the place of out-of-hours care-a market simulation based on discrete choice analysis. Health Policy. 2012;106:284-90.

6. Thompson AGH, Sunol R. Expectations as determinants of patient satisfaction: concepts, theory and evidence. Int J Qual Health Care. 1995;7(2):127-41.

7. Lancaster KJ. A new approach to consumer theory. J Pol Econ. 1966; 74(2):132-57.

8. Amaya-Amaya M, Gerard K, Ryan M. Discrete choice experiments in a nutshell. In: Ryan M, Gerard K, Amaya-Amaya M, editors. Using discrete choice experiments to value health and health care. Dordrecht: Springer; 2008. p. 13-46.
9. Thurstone LL. A law of comparative judgement. Psychol Rev. 1927;34:273-86

10. McFadden D. In: Zarembka, editor. Conditional logit analysis of qualitative choice behavior; 1974. p. 105-42.

11. Lancsar E, Louviere J. Conducting discrete choice experiments to inform healthcare decision making: a User's guide. PharmacoEconomics. 2008; 26(8):661-78.

12. Mangham LJ, Hanson K, McPake B. How to do (or not to do)... designing a discrete choice experiment for application in a low-income country. Health Pol Plan. 2009;24:151-8.

13. Bridges JFP, Hauber AB, Marshall D, et al. Conjoint analysis applications in health - a checklist: a report of the ISPOR good research practices for conjoint analysis task force. Value Health. 2011;4:403-13.

14. Donabedian A. Evaluating the quality of medical care. Milbank Q. 1966;44:166-203.

15. Donabedian A. The quality of care. How can it be assessed? JAMA. 1988; 260(12):1744-8.

16. Cheraghi-Sohi S, Hole AR, Mead N, et al. What patients want from primary care consultations: a discrete choice experiment to identify patients' priorities. Ann Fam Med. 2008;6:107-15.

17. Gerard K, Salisbury C, Street D, et al. Is fast access to general practice all that should matter? A discrete choice experiment of patients' preferences. J Health Serv Res Policy. 2008;13:3-10.

18. Gerard $K$, Lattimer $V$, Surridge $H$, et al. The introduction of integrated out-ofhours arrangements in England: a discrete choice experiment of public preferences for alternative models of care. Health Expect. 2006:9:60-9.

19. Gerard K, Tinelli M, Latter S, et al. Valuing the extended role of prescribing pharmacist in general practice: results from a discrete choice experiment. Value Health. 2012;15:699-707.

20. Gerard K, Tinelli M, Latter S, et al. Patients' valuation of the prescribing nurse in primary care: a discrete choice experiment. Health Expect. 2014;18:2223-35.

21. Hole AR. Modelling heterogeneity in patients' preferences for the attributes of a general practitioner appointment. J Health Econ. 2008;27:1078-94.

22. Lagarde M, Erens B, Mays N. Determinants of the choice of GP practice registration in England: evidence from a discrete choice experiment. Health Policy. 2015;119:427-36.

23. Rubin G, Bate A, George A, et al. Preferences for access to the GP: a discrete choice experiment. Br J Gen Pract. 2006;56:743-8.

24. Turner D, Tarrant C, Windridge $K$, et al. Do patients value continuity of care in general practice? An investigation using stated preference discrete choice experiments. J Health Serv Res Policy. 2007;12:132-7.

25. Caldow J, Bond C, Ryan M, et al. Treatment of minor illness in primary care: a national survey of patient satisfaction, attitudes and preferences regarding a wider nursing role. Health Expect. 2006;10:30-45.

26. Tinelli M, Ryan M, Bond C. Patients' preferences for an increased pharmacist role in the management of drug therapy. Int J Pharm Pract. 2009;17:275-82.

27. Mengoni A, Seghieri C, Nuti S. Heterogeneity in preferences for primary care consultations: results from a discrete choice experiment. Int J Stat Med Res. 2013;2:67-75.

28. Seghieri C, Mengoni A, Nuti S, et al. Applying discrete choice modelling in a priority setting: an investigation of public preferences for primary care models. Eur J Health Econ. 2014;15:773-85.

29. Pedersen LB, Kjaer T, Kragstrup J, et al. Do general practitioners know patients' preferences? An empirical study on the agency relationship at an aggregate level using a discrete choice experiment. Value Health. 2012;15:514-23.

30. Hjelmgren J, Anell A. Population preferences and choice of primary care models: a discrete choice experiment in Sweden. Health Policy. 2007;83:314-22.

31. Ahmed A, Fincham JE. Physician office vs retail clinic: patient preferences in care seeking for minor illnesses. Ann Fam Med. 2010;8:117-23.

32. Ahmed A, Fincham JE. Patients' view of retail clinics as a source of primary care: boon for nurse practitioners? J Am Acad Nurse Pract. 2011;23:193-9.

33. Tinelli M, Nikoloski Z, Kumpunen S. Decision-making criteria among European patients: exploring patient preferences for primary care services. Eur J Pub Health. 2014;25:3-9.

34. Coast J, Horrocks S. Developing attributes and levels for discrete choice experiments using qualitative methods. J Health Serv Res Policy. 2007;12:25-30.

35. Muehlbacher AC, Johnson FR. Choice experiments to quantify preferences for health and healthcare: state of the practice. Appl Health Econ Health Policy. 2016;14(3):253-66.

36. Veldwijk J, Lambooij MS, De Bekker-Grob EW, et al. The effect of including an opt-out option in discrete choice experiments. PLoS One. 2014;9:1-9. 